\title{
Reconfiguration of Distribution Networks with Distributed Generation Using a Dual Hybrid Particle Swarm Optimization Algorithm
}

\author{
Caoyuan Ma, ${ }^{1,2}$ Chunxiao Li, ${ }^{1,2}$ Xuezi Zhang, ${ }^{1,2}$ Guoxin Li, ${ }^{1,2}$ and Yonggang Han ${ }^{1,2}$ \\ ${ }^{1}$ School of Electrical and Power Engineering, China University of Mining and Technology, Xuzhou, Jiangsu 221116, China \\ ${ }^{2}$ Jiangsu Province Laboratory of Electrical and Automation Engineering for Coal Mining, China University of Mining and Technology, \\ Xuzhou, Jiangsu 221116, China \\ Correspondence should be addressed to Guoxin Li; li_guo_xin1@163.com
}

Received 23 December 2016; Accepted 5 July 2017; Published 6 August 2017

Academic Editor: Maria Patrizia Pera

Copyright (C) 2017 Caoyuan Ma et al. This is an open access article distributed under the Creative Commons Attribution License, which permits unrestricted use, distribution, and reproduction in any medium, provided the original work is properly cited.

\begin{abstract}
This paper proposes a reconfiguration strategy of distribution network with distribution generation (DG) based on dual hybrid particle swarm optimization algorithm. By the network structure simplification and branches grouping, network loss was selected as objective function, an improved binary particle swarm optimization algorithm (IBPSO) was used in branch group search, and the proposed group binary particle swarm optimization search algorithm was used in searching within the group to improve search efficiency and avoid early maturing. The proposed algorithm was tested on the IEEE 33-bus distribution power system and compared with other existing literature methods. The influence on the power flow of distribution network by DG position and capacity was studied. Simulation results illustrate that the proposed algorithm can get the optimal configuration results and significantly reduce system energy losses with fast convergence rate. In order to control the smart grid, using a dual hybrid particle swarm optimization algorithm to reconstruct a model, the result of simulation verifies the validity of the model. At the same time, the distributed power grid after reconstruction after optimization can effectively reduce the network loss and improve power supply quality.
\end{abstract}

\section{Introduction}

As clean and renewable energy sources, DG (distributed generation), such as photo voltaic and wind power, serves as mostly distributed and independent small power supply installed near the load or user $[1,2]$. With the powerful support by governments and the development of smart grid construction, the application of distributed power technology in power grids has been vigorously promoted.

Distributed power supply is a small generating unit directly arranged in distribution networks or users nearby to meet the specific requirements and support economic operation of distribution networks with environmental compatibility. And its power ranges from a few kilowatts to fifty megawatts [3-5]. Reconfiguration of distribution network refers to the change of network switch combination and adjustment of the structure of network operation by closing or opening the disconnected sectional switches and tie switches with constraints satisfied [4-6]. Its purpose is to prevent overload of transmission lines, balance the equilibrium electricity of users, reduce the power loss of the system, and maintain a good electrical quality. The optimization of the network with DG is intrinsically a multiple objective, noncontinuous, and multiple stage hybrid searching process where subgoals and constraints are mutually restricted, resulting in a kind of Nondeterministic Polynomial problem in mathematics [7]. The grid-connected of DG has a great influence on the distribution network, including the voltage distribution, power flow distribution, network line loss, power quality, and short circuit current, which cannot be ignored $[8,9]$.

The performance and efficiency of distribution network reconfiguration are largely dependent on an efficient search algorithm. Particle swarm optimization algorithm (PSO) is a swarm intelligence optimization algorithm. The principle of PSO is simple, robust, easy to achieve, and so on [10]. 
This paper proposes a reconfiguration strategy of distribution network with DG. The influences on power flow and voltage quality by the location, capacity, quantity, and type of the DG were analyzed. The improvements of particle swarm optimization algorithm and the static reconfiguration method were also analyzed. Distribution network simplification with DG was studied.

\section{Problem Representation}

Based on certain constraints, distribution network reconfiguration is to ensure that the network topology is radial and determine the distribution network achieving a certain indicator or a number of indicators to achieve the best operation state of the distribution network [11].

Distribution Network Reconfiguration with DG is a nonlinear, multiobject, discrete, multistage planning problem $[12,13]$. In this paper the objective function is to minimize active power loss of the whole distribution network.

$$
\min f=\sum_{i=1}^{N} K_{i} R_{i} \frac{P_{i}^{2}+Q_{i}^{2}}{U_{i}^{2}} .
$$

$i$ is branch number, $N$ is total branch numbers, $K_{i}$ is the state of switch ( 0 means open state and 1 means close state), $R_{i}$ is the resistor of branch $i, P_{i}$ and $Q_{i}$ are the active and reactive power of branch $i$, and $U_{i}$ is the up-layer node voltage of branch $i$.

The constraint conditions are as follows.

\section{(1) Power Flow Constraint Conditions}

$$
\begin{gathered}
P_{i}+P_{\mathrm{DG} i}=P_{D i}+U_{i} \sum_{j=1}^{N b} U_{j}\left(G_{i j} \cos \delta_{i j}+B_{i j} \sin \delta_{i j}\right) \\
Q_{i}+Q_{\mathrm{DG} i}=Q_{D i}+U_{i} \sum_{j=1}^{N b} U_{j}\left(G_{i j} \sin \delta_{i j}-B_{i j} \cos \delta_{i j}\right) .
\end{gathered}
$$

$P_{i}$ and $Q_{i}$ are the active and reactive power into node $i$, $P_{D i}$ and $Q_{D i}$ are active and reactive power of node $i$ load, $P_{\mathrm{DG} i}$ and $Q_{\mathrm{DG} i}$ are DG's active and reactive power into node $i, U_{i}$ and $U_{j}$ are voltage of nodes $i$ and $j, G_{i j}$ and $B_{i j}$ are admittance matrixes, and $\delta_{i j}$ is the phase difference between nodes $i$ and $j$.

(2) Voltage and Current Constraint Conditions

$$
\begin{gathered}
U_{i \min }<U_{i}<U_{i \max } \\
I_{i \min }<I_{i}<I_{i \max }
\end{gathered}
$$

\section{(3) Branch Active Power Constraint Condition}

$$
\left|P_{i j}^{\text {line }}\right|<\left|P_{i j, \max }^{\text {line }}\right| \text {. }
$$

$P_{i j}^{\text {line }}$ is actual active power between nodes $i$ and $j$, and $P_{i j \text {, max }}^{\text {line }}$ is the maximum active power between nodes $i$ and $j$.
(4) DG Power Constraint Conditions

$$
\begin{gathered}
P_{\mathrm{DG}, \min }<P_{\mathrm{DG}, i}<P_{\mathrm{DG}, \max } \\
Q_{\mathrm{DG}, \min }<Q_{\mathrm{DG}, i}<Q_{\mathrm{DG}, \max } .
\end{gathered}
$$

$P_{\mathrm{DG}, i}$ and $Q_{\mathrm{DG}, i}$ are active and reactive power of DG $i$.

(5) Network Topological Constraint. The network topology of the distribution network is radial.

\section{(6) Branch Capacity Constraint}

$$
\left|S_{l}\right| \leq S_{l, \max }
$$

$S_{l, \text { max }}$ is the maximum value for the complex power injection branch $l$. This is the branch capacity constraint condition.

\section{Distribution Network Simplification}

3.1. Analysis of the Radial Power Distribution Network. To ensure the high quality electrical energy in faults state, the distribution network was designed as a ring structure, which performs radial topology on grid operation state [14]. Due to the complexity of the distribution network reconfiguration, there are plenty of switch state combinations, leading to "ring" or "island" scenario that will damage the distribution network power supply and lose electrical power charge [15].

The isolated island is a subsystem which is not connected with the root node of the distribution system and is separated from the main power grid. The ring network means that there are a number of paths from the root node, resulting in a loop in the distribution system. The existence of isolated island or ring network shows that the switch combination is a unfeasible solution. Excessive unfeasible solutions can restrain optimization speed and reduce the efficiency of the search process of the reconfiguration algorithm $[16,17]$.

In this paper, the matrix method is adopted to judge the radial topological condition of distribution network. The main definition and theorem are as follows.

Definition 1. Set $n$ order simple directed graph as $G=\langle V, E\rangle$, $V=\left\{v_{1}, v_{2}, \ldots, v_{n}\right\}$. In the directed graph, $V$ is the node set and $E$ is the edge set. Define a matrix $P=\left[P_{i j}\right]_{n \times n}$ and assume

$$
P_{i j}= \begin{cases}1 & v_{i} \text { can reach } v_{j} \\ 0 & v_{i} \text { cannot reach } v_{j} .\end{cases}
$$

Then we call the matrix $P$ as the reachable matrix of graph $G$. The reachable matrix shows whether there is a path between any two nodes in the distribution network.

Theorem 2. If there is a directed graph $G=\langle V, E\rangle$ with adjacent matrix,

$$
A=\left|\begin{array}{l}
a_{11}, a_{12}, \ldots, a_{1 n} \\
a_{21}, a_{22}, \ldots, a_{2 n} \\
\ldots \ldots \ldots . . . \\
a_{n 1}, a_{n 2}, \ldots, a_{n n}
\end{array}\right|
$$




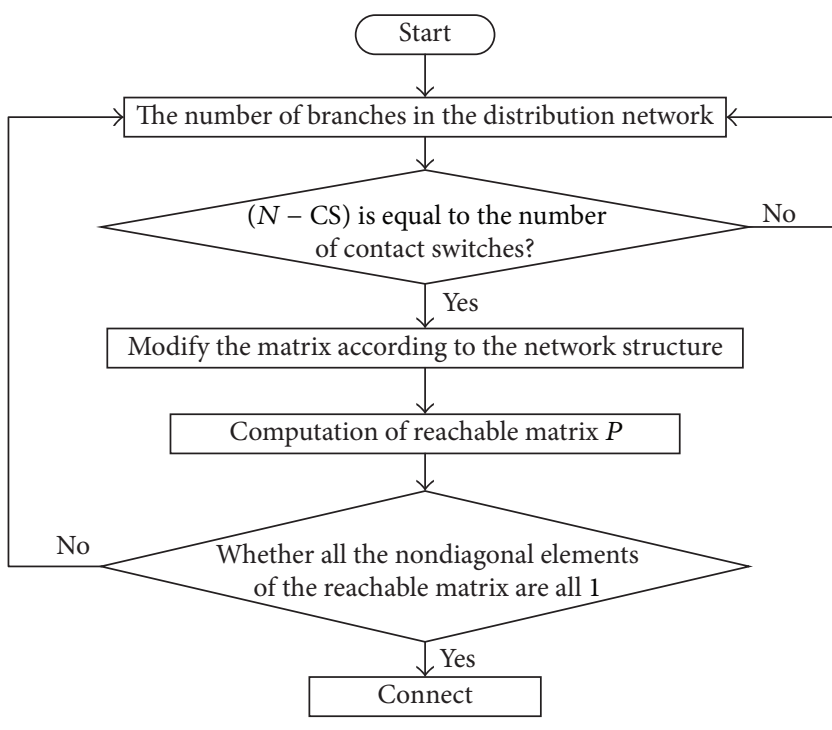

FIGURE 1: Flow chart of radial distribution network analysis.

Then, it can be concluded that the reachable matrix of the network is $P=A(+) A^{2}(+) A^{3}(+) \cdots(+) A^{n}$, where $(+)$ is the Boolean sum.

Theorem 3. Suppose $G=\langle V, E\rangle$ is a simple directed graph with no self-loop and $P$ is its reachable matrix. Then the necessary and sufficient condition for the strong connectivity of the graph $G$ is that the elements of $P$ are 1 except diagonal elements.

The indirect graphs can be seen as a special case of directed graph when every side in indirect graph is equivalent to two sides which have opposite directions. Therefore, the connectivity of indirect graphs can be identified by the strong connection criterion of directed graphs. The distribution system is radial network only when the number of rings is equal to the number of the contact switches. That is, the number of disconnected branches is $N-\mathrm{CS}=$ (total network branch $)-($ effective node number $)+1$ [18]

The flow chart to identify the connectivity of distribution network is shown in Figure 1.

3.2. Distribution Network Simplification. In the reconfiguration of distribution network, the value of the particle position in every dimension is random between 0 and 1 , resulting in a large number of unfeasible solutions. Therefore, it is necessary to reduce the particle encoding dimension, simplify the structure of the distribution network, and improve the search efficiency. The IEEE 33 nodes network was shown in Figure 2(a). The network can be simplified according to the rules as follows: the branches with the nodes whose degree (the number of edges adjacent to the node) is 2 is merged into a group and numbered, and the number greater than 2 is retained, as shown in Figure 2(b). Then renumber nodes and branches, as shown in Figure 2(c). Each branch group contains a number of branches; for example, the branch group 6 was composed of the branches 12-13, 13-14, and 14-15. Hence the coding dimension is greatly reduced and the dimension of solution vector is simplified from 37 to 13. Many unfeasible solutions were avoided and efficiency of the algorithm was improved.

In addition, assuming that there are $M$ branches in the network topology, the particle optimization coding dimension of the branch groups is $M$. Each particle code is a vector composed of 0 and 1, which represents a kind of topology structures of distribution network.

And the particle encoding needs to satisfy

$$
N-\mathrm{CS}=N T-N E+1
$$

$N T$ is total number of branches, and NE is effective node number.

In this way, the number of branch groups disconnected in the reconfiguration of distribution network with DG is the basic mesh number. When we break a branch which belongs to a disconnected branch group, it must satisfy the radial topological condition, avoid the production of the infeasible solutions, and thus improve the search efficiency of the algorithm.

\section{Improved Binary Particle Swarm Optimization Algorithm (BPSO)}

4.1. Particle Swarm Optimization. Particle Swarm Optimization (PSO) is a kind of global optimization algorithm based on social group behavior, which is derived from the simulation of bird foraging process [19].

Suppose a population consists of $n$ particles, which is generally 20 in the distribution network reconfiguration. Each particle in the population is searching for optimal solutions in a $m$-dimensional space with a finite speed as the real problem solution is $m$-dimension [20]. The particle of $j$ in the population is composed of three $m$-dimensional vectors, that is, respectively, the individual optimal location in history $p_{j}=\left(p_{j 1}, p_{j 2}, \ldots, p_{j m}\right)$, the current position $x_{j}=$ $\left(x_{j 1}, x_{j 2}, \ldots, x_{j m}\right)$, and particle velocity $v_{j}=\left(v_{j 1}, v_{j 2}, \ldots\right.$, $\left.v_{j m}\right) i=1,2, \ldots, n$.

The position of each particle will be calculated and evaluated before next iteration according to the actual problem represented. If the current position is superior to the individual or the best record of population position in history, this position will be deemed as the best position. Otherwise, the location will not be updated.

For each individual in the population, the iteration formula of the velocity and position is

$$
\begin{aligned}
v_{j m}^{k+1}= & w v_{j m}^{k}+c_{1} \operatorname{rand}\left(p_{j m}^{k}-x_{j m}^{k}\right) \\
& +c_{2} \operatorname{rand}\left(g_{j m}^{k}+x_{j m}^{k}\right) \\
x_{j m}^{k+1}= & x_{j m}^{k}+v_{j m}^{k+1},
\end{aligned}
$$

where $c_{1}$ and $c_{2}$ are the individual learning factor and social learning factor. Their values are nonnegative and range from 0 to 4 , which are usually assigned to 2 . They can approach to the global optimum by learning by themselves and the experience 


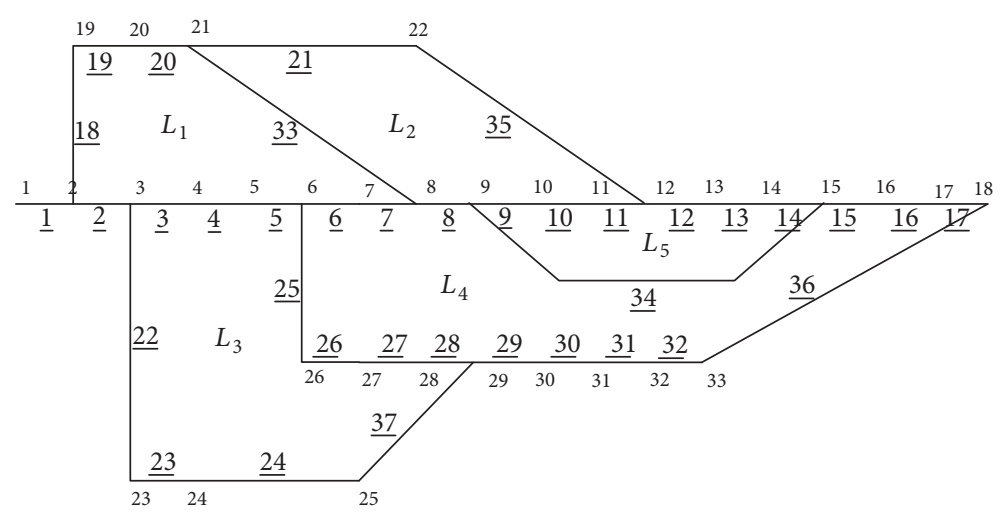

(a) IEEE 33 nodes network

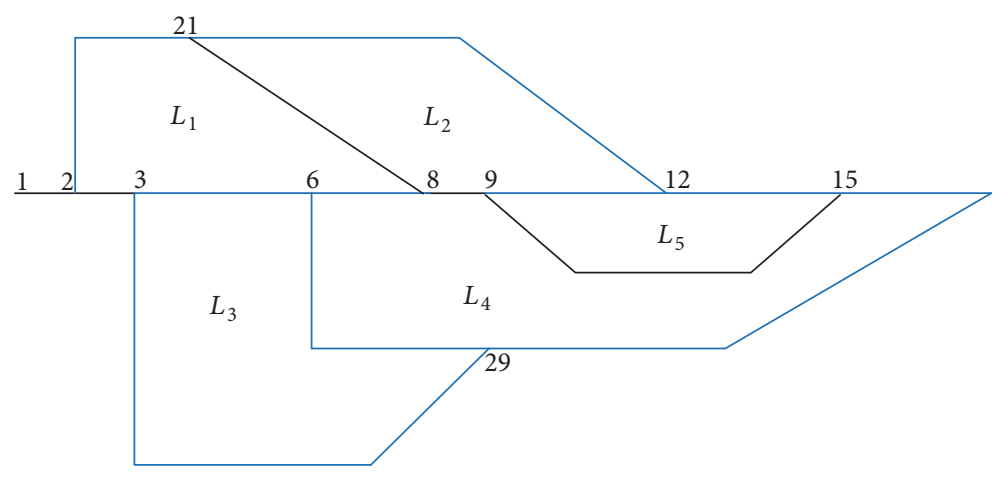

(b) Network structure after branches simplification

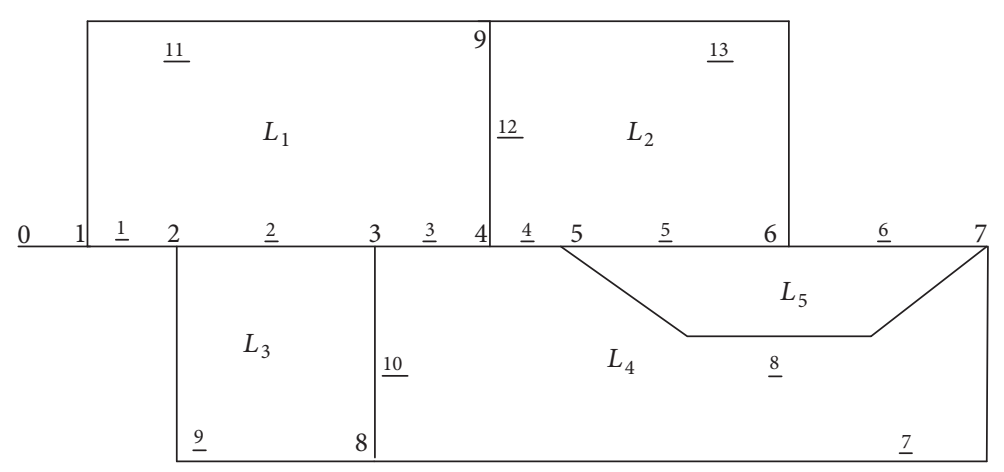

(c) Network structure after renumbering nodes and branches

FIGURE 2: Sketch of distribution network simplification.

of the population. rand is a uniform random number between $[0,1] . v_{\max }$ is the maximum velocity of the particle, and the range of $v_{\max }$ is $\left[-v_{\max }, v_{\max }\right]$, which is usually the range of particle value per dimension.

\subsection{Improved Binary Particle Swarm Optimization Algorithm} (IBPSO). Compared with the continuous PSO, the velocity update formula of binary particle swarm optimization algorithm (BPSO) is the same as that of the former, but the position update formula is different. During the initialization of BPSO particle population, the solution of problem is transformed into binary code [13].
In BPSO, the position change of the particle is the probability that the velocity of each dimension is converted to the bit value 1 . Assuming $v_{i m}^{k}$ is the velocity of particle $i$, the $m$ th is dimension of the $k$ th generation, and the probability of the bit value 1 is $s\left(v_{i m}^{k}\right)$, so the probability of the bit value 0 is $1-s\left(v_{i m}^{k}\right)$. Therefore, if the $m$ th dimension and $(k-1)$ th generation of the $i$ th particle is 1 , then the probability of the occurrence of the $m$ th and $k$ th generation is $1-s\left(v_{i m}^{k}\right)$. Similarly, if the $m$ th dimension and $(k-1)$ th generation of the $i$ th particle is 0 , then the probability of the occurrence of the $m$ th dimension and $k$ th generation is $s\left(v_{i m}^{k}\right)$. If the $m$ th dimension and $(k-1)$ th generation of the $i$ th particle 
is $s\left(v_{i m}^{k-1}\right)$, then the probability of 0 is $1-s\left(v_{i m}^{k-1}\right)$. So the probability of occurrence of the $m$ th dimension and $k$ th generation of the $i$ th particle is

$$
p_{i m}^{k}=s\left(v_{i m}^{k-1}\right)\left(1-s\left(v_{i m}^{k}\right)\right)+\left(1-s\left(v_{i m}^{k-1}\right)\right) s\left(v_{i m}^{k}\right)
$$

According to (12), the probability of particle $i$ change, the $m$ th dimension and $k$ th generation, is related to the velocity of the two generations. When the particles' velocities of both generations are close to 0 , the probabilities of bit value change and the probability of random search are probable. The probability of particles convergence to the global optimal position is very small. Expression (10) is composed of three parts. Firstly, $w v_{j m}^{k}$ is the velocity inertia showing the impact of the particle's history velocity on its current velocity. Secondly, $c_{1} \operatorname{rand}\left(p_{j m}^{k}-x_{j m}^{k}\right)$ reflects the individual's self-learning ability, a part of "self-cognition." Thirdly, $c_{2} \operatorname{rand}\left(g_{j m}^{k}-v_{j m}^{k}\right)$ reflects the individual's ability to learn from the population, a part of "social cognition." Research results show that when $\left(p_{j m}^{k}-\right.$ $\left.x_{j m}^{k}\right)$ and $\left(g_{j m}^{k}-x_{j m}^{k}\right)$ are 0,1 , and -1 , respectively, 0 means that the values of $p_{j m}^{k} g_{j m}^{k}$ and $x_{j m}^{k}$ are equal; 1 means that $p_{j m}^{k}$ or $g_{j m}^{k}$ is 1 , while the value of $x_{j m}^{k}$ is $0 ;-1$ means that $p_{j m}^{k}$ or $g_{j m}^{k}$ is 0 , while the value of $x_{j m}^{k}$ is 1 . In the latter part of particle swarm search, when $v_{j m}^{k}$ is close to 0 , the position of the particle is close to the optimal particle population and the optimal solution, and the particle value should remain intact. When $v_{j m}^{k}<0$, the value of the historical optimal solution of the optimal particle in population is equal to 0 . At this point, the probability of the particle position is 1 , and the position of the particle should be inverted to 0 . When $v_{j m}^{k}>0$, the value of the historical optimal solution for the population of the optimal particle equals reversely 1 .

The relationship between the particle position value and the optimal particle position of the population, according to the above method, can balance the local and global search ability at the later stage and make the result tend to the optimal particle of population and converge to the global optimal particle eventually.

\section{Improvements of the Original BPSO}

(1) If Iteration $<\lambda \times$ MaxIter, the velocity and position of particles will be updated. If Iteration $\geq \lambda \times$ MaxIter, according to (13), (14), and (15), the velocity and position will be updated.

(2)

$$
s\left(v_{j m}^{k}\right)= \begin{cases}1-\frac{2}{1+\exp \left(-v_{j m}^{k}\right)} & v_{j m}^{k} \leq 0 \\ \frac{2}{1+\exp \left(-v_{j m}^{k}\right)}-1 & v_{j m}^{k}>0 .\end{cases}
$$

When $v_{j m}^{k} \leq 0$,

$$
x_{j m}^{k}= \begin{cases}0 & \operatorname{rand}() \leq s\left(v_{j m}^{k}\right) \\ x_{j m}^{k} & \text { otherwise. }\end{cases}
$$

When $v_{j m}^{k}>0$,

$$
x_{j m}^{k}= \begin{cases}1 & \text { rand }() \leq s\left(v_{j m}^{k}\right) \\ x_{j m}^{k} & \text { otherwise. }\end{cases}
$$

$\lambda \in[0,1]$. Iteration is the number of iterations, and its maximum value is MaxIter.

IBPSO and the standard BPSO are different in the probability mapping function. The goal of the new probability mapping function is that the probability mapping function value is 0 when the velocity tends to 0 , so the local optimization efficiency is enhanced. Then, by new probability function, the particle's bit value remains intact when it approximately equals 0 . When $V<0$, the position of the particle is set to 0 . And when $V>0$, it is set to 1 . When the velocity is 0 , the probability of particle's position change is close to 0 , and its local search ability is enhanced. By this way, the particle swarm was converged to global optimal particle finally.

\section{Reconfiguration of Distribution Network with DG}

5.1. Distribution Network Reconfiguration Based on Dual Hybrid Particle Swarm Optimization Algorithm. To finish the distribution network reconfiguration, distribution network simplification, branch groups selection and optimization, branches selection and optimization in branch group, and power flow calculation under constraint conditions, dual hybrid particle swarm optimization algorithm is proposed in this paper. IBPSO was used in branch group search, and the proposed group BPSO was used in searching within the group. This is reconfiguration of distribution network by dual hybrid particle swarm optimization algorithm.

Take the IEEE 33 node distribution system as an example. Firstly, determine the matrix $A$, which is the relationship between branch group number and branch within group number, as shown in (16). Secondly, use 0 or 1 to describe branches' states within the group (open or closed) and set the vector of each branch group, as shown in (17). There is only one branch disconnected in every group when updating the speed and location. Thirdly, based on the disconnected branch group number, select the disconnected branch within group. The speed updating formula is given as (18) and the location formula is the same as before. 


$$
\begin{aligned}
& A=\left[\begin{array}{cccccccc}
2 & 0 & 0 & 0 & 0 & 0 & 0 & 0 \\
3 & 4 & 5 & 0 & 0 & 0 & 0 & 0 \\
6 & 7 & 0 & 0 & 0 & 0 & 0 & 0 \\
8 & 0 & 0 & 0 & 0 & 0 & 0 & 0 \\
9 & 10 & 11 & 0 & 0 & 0 & 0 & 0 \\
12 & 13 & 14 & 0 & 0 & 0 & 0 & 0 \\
15 & 16 & 17 & 29 & 30 & 31 & 32 & 36 \\
34 & 0 & 0 & 0 & 0 & 0 & 0 & 0 \\
22 & 23 & 24 & 37 & 0 & 0 & 0 & 0 \\
25 & 26 & 27 & 28 & 0 & 0 & 0 & 0 \\
18 & 19 & 20 & 0 & 0 & 0 & 0 & 0 \\
33 & 0 & 0 & 0 & 0 & 0 & 0 & 0 \\
21 & 35 & 0 & 0 & 0 & 0 & 0 & 0
\end{array}\right] \\
& A 1=[0] \text {; } \\
& A 2=[1,0,1] ; \\
& A 3=[0,1] \text {; } \\
& A 4=[0] \text {; } \\
& A 5=[1,1,0] \text {; } \\
& A 6=[0,1,1] \text {; } \\
& A 7=[1,1,1,1,0,1,1,1] ; \\
& A 8=[0] \text {; } \\
& A 9=[1,0,1,1] \text {; } \\
& A 10=[1,1,0,1] ; \\
& A 11=[1,0,1] ; \\
& A 12=[0] \text {; } \\
& A 13=[1,0] ; \\
& v_{i m r}^{k+1}= \begin{cases}w v_{i m r}^{k}+c_{1} \operatorname{rand}\left(p_{i m r}^{k}-x_{i m r}^{k}\right)+c_{2} \operatorname{rand}\left(g_{i m r}^{k}-x_{i m r}^{k}\right) & x_{i m}^{k}=0, p_{i m}^{k}=g_{i m}^{k}=0 \\
w v_{i m r}^{k}+c_{1} \operatorname{rand}\left(p_{i m r}^{k}-x_{i m r}^{k}\right)+c_{2} v_{\max }(2 \operatorname{rand}-1) & x_{i m}^{k}=0, p_{i m}^{k}=0, g_{i m}^{k}=1 \\
w v_{i m r}^{k}+c_{1} v_{\max }(2 \operatorname{rand}-1)+c_{2} \operatorname{rand}\left(g_{i m r}^{k}-x_{i m r}^{k}\right) & x_{i m}^{k}=0, p_{i m}^{k}=1, g_{i m}^{k}=0 \\
w v_{i m r}^{k}+c_{1} v_{\max }(2 \operatorname{rand}-1)+c_{2} v_{\max }(2 \operatorname{rand}-1) & x_{i m}^{k}=0, p_{i m}^{k}=1, g_{i m}^{k}=1 \\
v_{i m r}^{k} & x_{i m}^{k}=1,\end{cases}
\end{aligned}
$$

where $v_{i m r}^{k+1}$ is the speed of particle $i$ at $k+1$ th iteration, which in the branch of group $m, p_{i m r}^{k}$ and $g_{i m r}^{k}$ are particles' history optimal positions and population optimal position, and $x_{i m r}^{k}$ is connection condition of the branch $r$ in group $m$ at $k$ th iteration.
5.2. Reconfiguration Flow of Distribution Network with DG. The IBPSO and BPSO in branch group are core algorithms in distribution network reconfiguration. The branch selection is optimized by IBPSO, while the branch group selection is optimized by BPSO within group. The detailed steps and the 


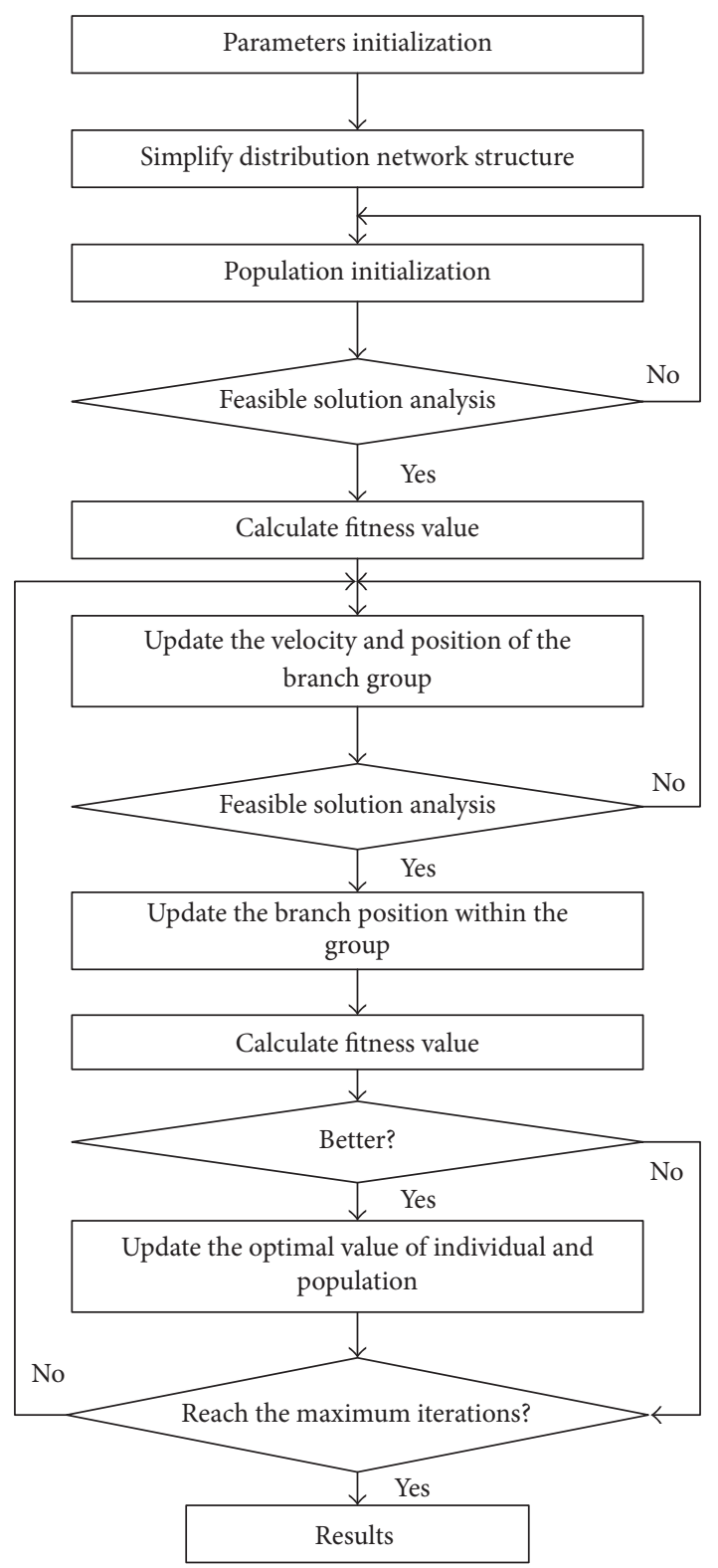

Figure 3: Flow chart of distribution network reconfiguration algorithm.

specific implementation flow chart of network reconfiguration algorithm are shown in Figure 3.

Step 1. Set the values of particle population number $N$, the number of groups, and all other parameters.

Step 2. According to the simplified structure of distribution network, the nodes and branches of the distribution network are renumbered. Set the internal branch number within branch group, such as the column number of the elements in a matrix $A$. Then, the particle dimension of branch group $D$ and dimension of branches within group $D^{\prime}$ are determined, and then parameters of the population particles are initialized.
TABLE 1: Reconfiguration results without DG connected into grid.

\begin{tabular}{lccc}
\hline $\begin{array}{l}\text { Reconfiguration } \\
\text { condition }\end{array}$ & $\begin{array}{c}\text { Disconnect } \\
\text { network switch }\end{array}$ & $\begin{array}{c}\text { Minimum } \\
\text { node voltage }\end{array}$ & $\begin{array}{c}\text { Network } \\
\text { loss/kW }\end{array}$ \\
\hline $\begin{array}{l}\text { Before } \\
\text { reconfiguration }\end{array}$ & $\begin{array}{c}8-21 \text { 9-15 12-22 } \\
18-3325-29\end{array}$ & 0.9185 & 202.65 \\
Literature & $\begin{array}{c}7-89-1014-15 \\
28-2932-33\end{array}$ & 0.9412 & 139.90 \\
$\begin{array}{l}\text { Algorithm in this } \\
\text { paper }\end{array}$ & $\begin{array}{c}7-89-1014-15 \\
25-2932-33\end{array}$ & 0.9378 & 139.47 \\
\hline
\end{tabular}

Step 3. Update the speed and position of the branch group. If the structure of the distribution network is radial, go to the next step; otherwise, repeat this step.

Step 4. Update disconnected branch location within the group branch organization. According to the matrix $A$, find the power flow calculation in actual disconnected branch number and calculate its fit value.

Step 5. Compare the particle updated fitness value with the optimal particle fitness value in history, as well as the particle swarm optimum fitness value. If the current position is better, the value of current position will be selected as the particle's best individual historical fitness value and the particle swarm optimum fitness value. This position will be selected to update the best position of individual history and the best position of population in history.

Step 6. If the iteration number is less than the predetermined, go back to Step 3.

Step 7. If the iteration number reaches the predetermined, the optimum reconfiguration results are obtained and the program ends.

\section{Case Studies}

The proposed algorithms are used on the IEEE 33 node distribution system to verify the validity, convergence, and stability. IEEE 33 nodes network structure was shown in Figure 2(a). Assuming the particle population $N=20$, the loop termination condition is

$$
\max \left|U^{k}(i)-U^{k-1}(i-1)\right| \leq 0.000001 .
$$

As shown in Table 1, the active power loss of the network is $139.47 \mathrm{~kW}$ after reconfiguration. It is $31.18 \%$ lower than the active power loss before reconfiguration which is $202.65 \mathrm{KW}$. As shown in Figure 4, the overall voltage of the reconfigured network is generally higher than that of the network before reconstruction. The minimum value of node voltage rose from 0.9185 P.U. to 0.9378 P.U.

DG nodes were considered as PQ nodes for computation purpose, as in Table 2.

Table 3 shows the reconfiguration results with DG connected into grid by the algorithm in this paper. The active power loss of the network is $112.58 \mathrm{~kW}$, which is $44.44 \%$ lower than active power loss before reconfiguration. As 


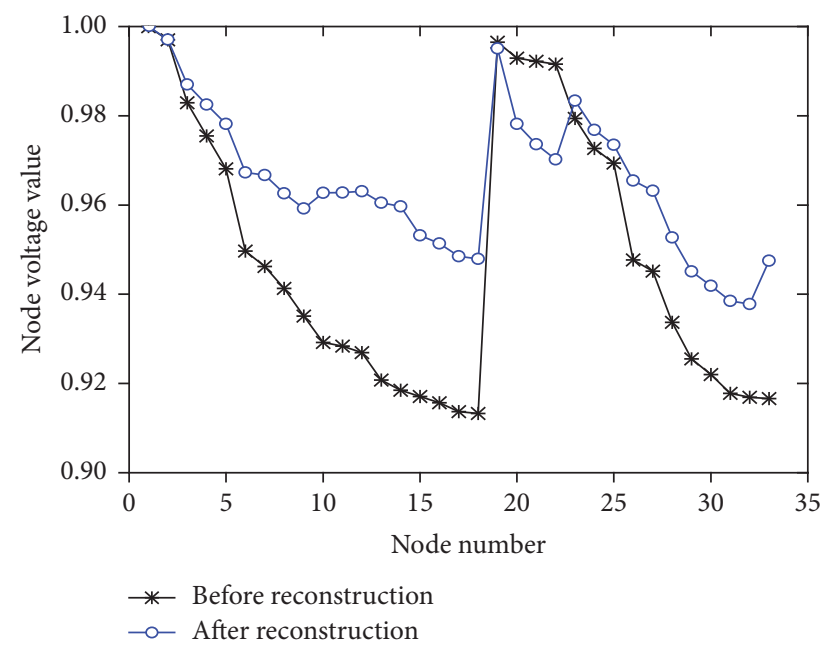

FIGURE 4: Voltage change tendency of nodes.

TABLE 2: Location and capacity of DG.

\begin{tabular}{lccc}
\hline $\begin{array}{l}\text { Distributed } \\
\text { power serial } \\
\text { number }\end{array}$ & Access point & $\begin{array}{c}\text { Active } \\
\text { power/kW }\end{array}$ & Power factor \\
\hline DG1 & 7 & 100 & 0.8 \\
DG2 & 9 & 50 & 0.9 \\
DG3 & 22 & 50 & 0.85 \\
DG4 & 25 & 250 & 0.9 \\
\hline
\end{tabular}

TABLE 3: Reconfiguration results with DG connected into grid.

\begin{tabular}{lccc}
\hline $\begin{array}{l}\text { Reconfiguration } \\
\text { condition }\end{array}$ & $\begin{array}{c}\text { Disconnect } \\
\text { network switch }\end{array}$ & $\begin{array}{c}\text { Minimum } \\
\text { node voltage }\end{array}$ & $\begin{array}{c}\text { Network } \\
\text { loss/kw }\end{array}$ \\
\hline $\begin{array}{l}\text { Before } \\
\text { reconfiguration }\end{array}$ & $\begin{array}{c}8-219-1512-22 \\
18-3325-29\end{array}$ & 0.9185 & 202.65 \\
$\begin{array}{l}\text { Before } \\
\text { reconfiguration } \\
\text { (DG connected) }\end{array}$ & $\begin{array}{c}8-219-1512-22 \\
18-3325-29\end{array}$ & 0.9194 & 169.50 \\
$\begin{array}{l}\text { Literature } \\
\text { 7-8 9-10 14-15 }\end{array}$ & 0.9482 & 115.04 \\
$\begin{array}{l}\text { Algorithm in this } \\
\text { paper }\end{array}$ & $\begin{array}{c}8-9532-33 \\
28-2932-33\end{array}$ & 0.9448 & 112.58 \\
\hline
\end{tabular}

shown in Figure 5, the overall voltage of the reconfigured network is generally higher than that of the network before reconstruction. The minimum value of node voltage rises from 0.9185 P.U. to 0.9448 P.U. The reconfiguration result proves the superiority of the proposed algorithm, which was suitable to the reconfiguration of Distribution Network with DG.

Figure 6 shows the active power loss convergence curve of the network, which shows that the network loss converges when the iteration number was greater than 3 . This proves that the proposed dual hybrid particle swarm optimization algorithm can quickly jump out of local optimal solution and converge to the optimal switch combination simultaneously.

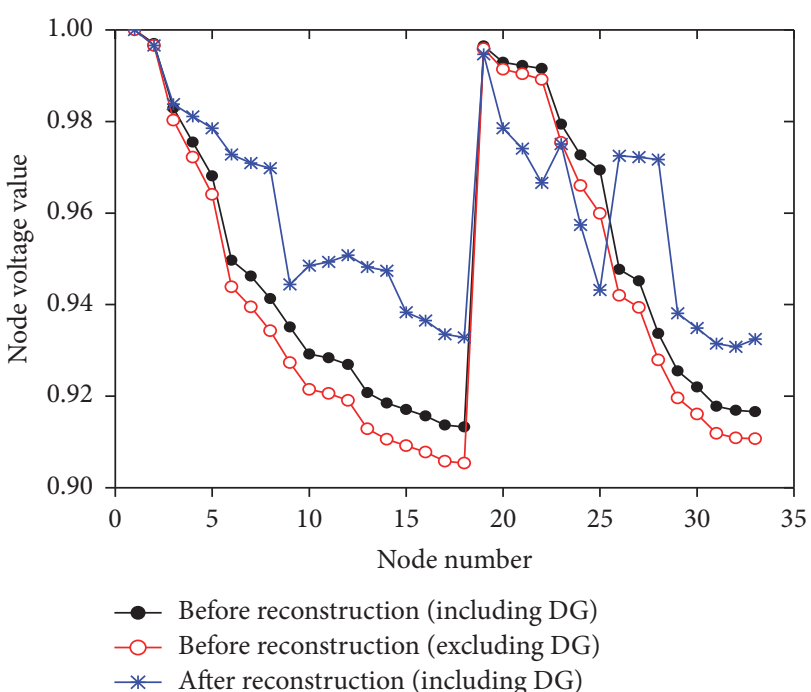

FIGURE 5: Nodes voltage change tendency with and without DG connected.

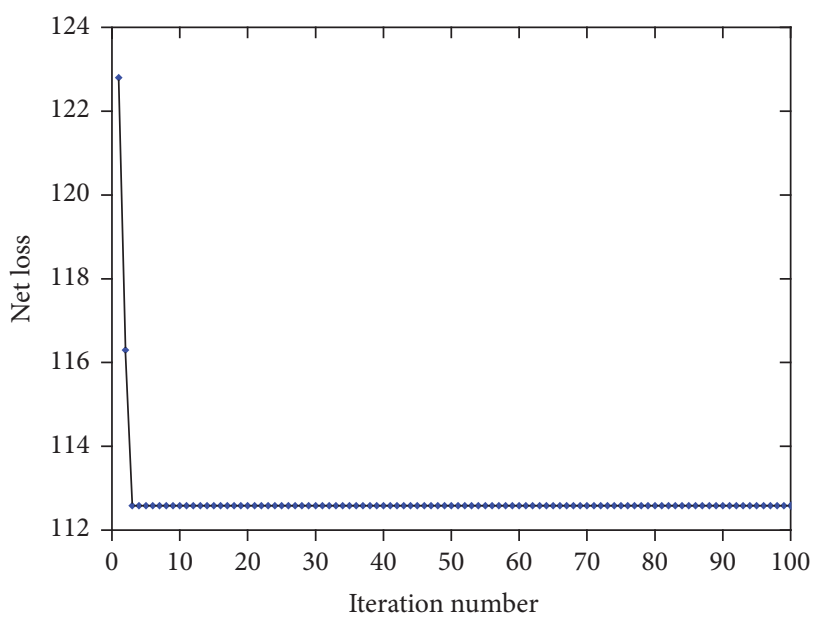

FIGURE 6: Loss convergence curve.

After 50 times simulations of the example by the computer with Windows 7 operating system, $2.20 \mathrm{GHz}$ Intel CPU, $4.0 \mathrm{G}$ memory, the results are shown in Figure 7, which shows the average number of iterations is 5 times, and the active power loss is about $112 \mathrm{~kW}$.

DG with different types and capacities are selected to verify the validity of the algorithm, as shown in Table 4.

Table 5 shows that active power loss of the network is $112.77 \mathrm{~kW}$ after reconfiguration. It is $44.35 \%$ lower than the active power loss of the network before reconfiguration which is $202.65 \mathrm{KW}$. The minimum value of node voltage increases from 0.9185 P.U. to 0.9380 P.U. Figure 8 shows that the overall voltage of the reconfigured network is generally higher than that of the network before reconstruction.

After 100 times simulations of the example, the results are shown in Figure 9, and the average number of iterations is 16 times. The above analysis results show that the economy of 
TABLE 4: Location and capacity of DG.

\begin{tabular}{lccc}
\hline Distributed power serial number & Access point & Distributed power type & Parameter \\
\hline DG1 & 4 & Doubly fed induction generator $(P Q)$ & $P=100 \mathrm{~kW}, Q=100 \mathrm{kVar}$ \\
DG2 & 17 & Photovoltaic cells $(P I)$ & $P=150 \mathrm{~kW}, I=10 \mathrm{~A}$ \\
DG3 & 25 & Fuel cell $(P V)$ & $P=200 \mathrm{~kW}, U=12.66 \mathrm{kV}$ \\
DG4 & 30 & Asynchronous fan $(P Q(V))$ & $P=150 \mathrm{~kW}, Q=100 \mathrm{kVar}$ \\
\hline
\end{tabular}

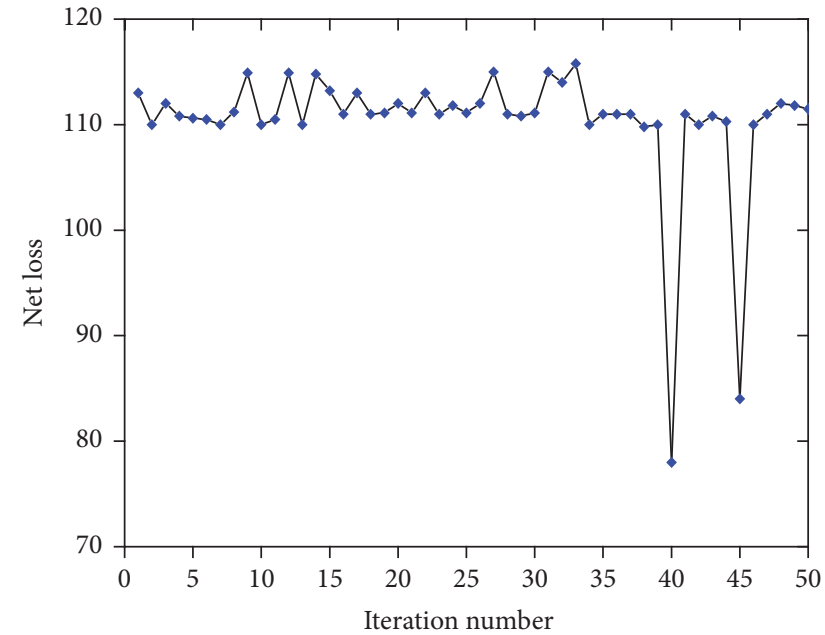

FIgURE 7: Stability analysis of double hybrid PSO method.

TABLE 5: Reconfiguration results with DG connected.

\begin{tabular}{lccc}
\hline $\begin{array}{l}\text { Reconfiguration } \\
\text { condition }\end{array}$ & $\begin{array}{c}\text { Disconnect } \\
\text { network switch }\end{array}$ & $\begin{array}{c}\text { Minimum } \\
\text { node voltage }\end{array}$ & $\begin{array}{c}\text { Network } \\
\text { loss/kW }\end{array}$ \\
\hline $\begin{array}{l}\text { Before } \\
\text { reconstruction }\end{array}$ & $\begin{array}{c}8-219-1512-22 \\
18-3325-29\end{array}$ & 0.9185 & 202.65 \\
$\begin{array}{l}\text { Before } \\
\text { reconfiguration } \\
\text { (including DG) }\end{array}$ & $\begin{array}{c}8-219-1512-22 \\
18-3325-29\end{array}$ & 0.9274 & 175.62 \\
$\begin{array}{l}\text { After } \\
\text { reconfiguration } \\
\text { (including DG) }\end{array}$ & $\begin{array}{c}11-1228-2930-31 \\
8-219-15\end{array}$ & 0.9380 & 112.77 \\
\hline
\end{tabular}

the system was improved, and the stability and reliability of the system were enhanced after reconfiguration.

\section{Summary}

Based on dual hybrid particle swarm algorithm combined with the network structure simplification and branches grouping, this paper deals with the problem of distribution systems reconfiguration. The proposed algorithm is tested on the IEEE 33-bus distribution power system and compared with other existing literature methods. The simulation results show that the proposed algorithm has a faster optimization speed for the distribution network with DG, which can not only reduce the network loss and support system voltage but also improve the economy and reliability of the system. The proposed algorithm was suitable to search for the best switch combination of distribution network with DG.

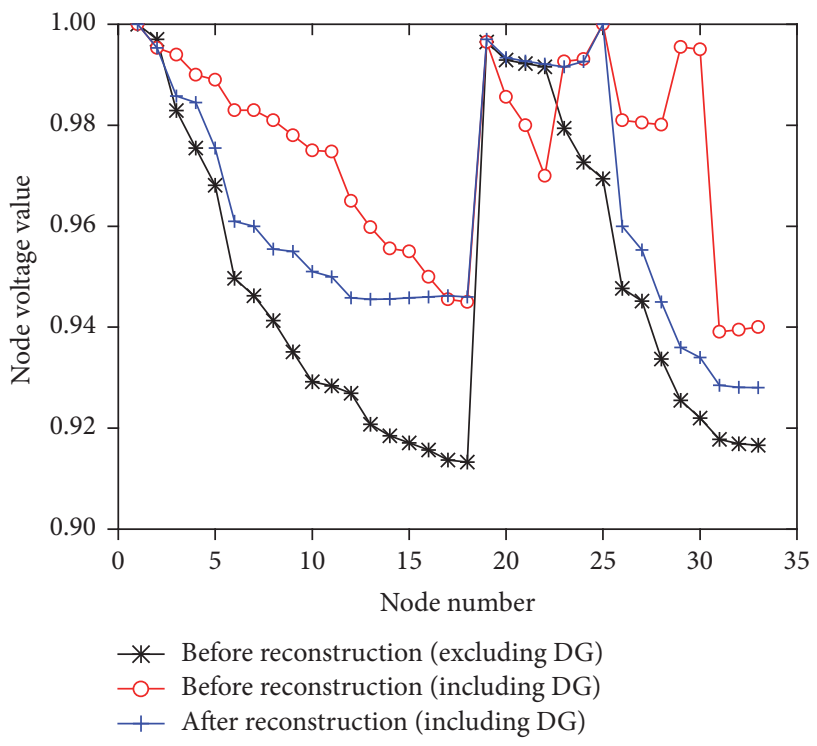

FIGURE 8: Nodes voltage with and without DG connected.

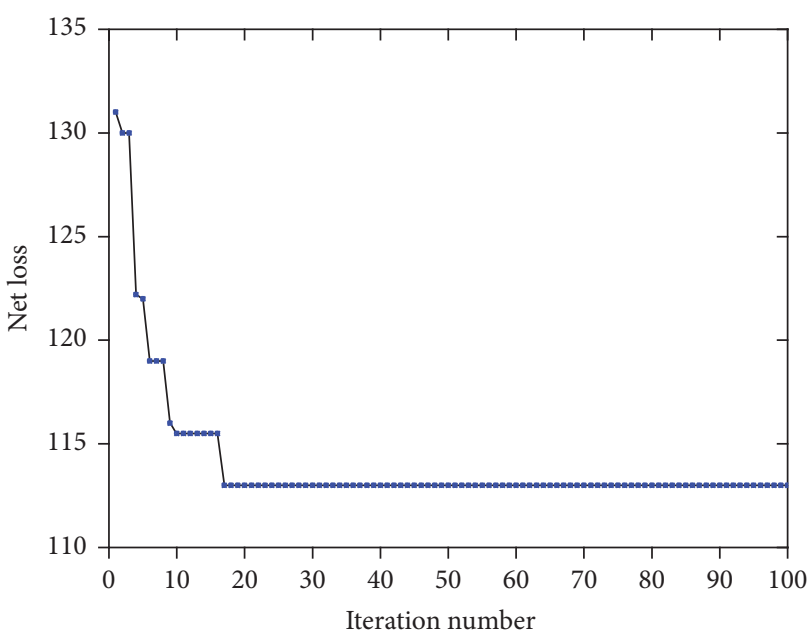

FIGURE 9: Loss convergence curve.

Distributed power grid connected is an inevitable trend with the development of smart city and modern power grid technology. It is of great significance for energy saving and emission reduction and improving energy structure. In this paper, the reconfiguration of smart grid with distributed generation is studied. From the simulation results, it can be seen that after the reconfiguration of the distributed power grid, it can effectively reduce the loss of distribution network, 
improve the quality of the power supply voltage, and improve the power quality of the grid. The method of research and development of this paper has certain reference function for the optimization of power grid configuration in the future.

\section{Conflicts of Interest}

The authors declare that there are no conflicts of interest regarding the publication of this paper.

\section{Acknowledgments}

This work is supported by the Jiangsu Provincial Natural Science Foundation of China (Grant no. BK20130187) and China Scholarship Council (CSC no. 201406425007).

\section{References}

[1] F. Z. Peng, "Special issue on distributed power generation," IEEE Transactions on Power Electronics, vol. 19, no. 5, pp. 1157-1158, 2004.

[2] E. M. Carreno, R. Romero, and A. Padilha-Feltrin, "An efficient codification to solve distribution network reconfiguration for loss reduction problem," IEEE Transactions on Power Systems, vol. 23, no. 4, pp. 1542-1551, 2008.

[3] W. El-Khattam and M. M. A. Salama, "Distributed generation technologies, definitions and benefits," Electric Power Systems Research, vol. 71, no. 2, pp. 119-128, 2004.

[4] W. Hongjian and X. Lei, "The dynamic reconfiguration of distribution network with wind turbine and electric vehicle based on genetic algorithm," Journal of Electrical Engineering, vol. 31, no. 2, pp. 197-220, 2016.

[5] W. Jiajia, L. Lin, L. Junyong et al., "Based on improved hierarchical forward and backward substitution method with distributed generation units of distribution network reconfiguration," Power System Technology, vol. 34, no. 3, pp. 109-112, 2010.

[6] C. Li, Q. Yang, Y. Wei, H. Luo, and Q. Zhang, "Distribution network reconfiguration method considering the correlation between wind speed and load," Dianli Zidonghua Shebei/Electric Power Automation Equipment, vol. 36, no. 2, pp. 148-153, 2016.

[7] X. Chen and J. weighted, "Application of the mutation particle swarm optimization algorithm in the vehicle routing problem," Computer And Digital Engineering, vol. 34, no. 9, pp. 19-21, 2006.

[8] T. Jen-Hao and M. L. Whe, "Current-based power flow solutions for distribution systems," ICPST Beijing, China, 1994: 414-418.

[9] R. D. Zimmerman and H.-D. Chiang, "Fast decoupled power flow for unbalanced radial distribution systems," IEEE Transactions on Power Systems, vol. 10, no. 4, pp. 2045-2052, 1995.

[10] Y. L. Mei, X. Mingxia, X. Jianjun et al., "Power flow algorithm for distribution network with distributed generation," Power System Protection And Control, vol. 41, no. 5, pp. 18-22, 2013.

[11] X. Xiang, D. Liu, N. Xiang, and B. Wang, "Distribution network reconfiguration based on parallel Tabu search algorithm," Power System Technology, vol. 36, no. 8, pp. 101-105, 2012.

[12] X. Lixiong, L. Lin, and L. Junyong, "Reconfiguration of distribution network based on improved particle swarm optimization algorithm," Automation of Electric Power Systems, vol. 30, no. 7, pp. 27-30, 2006.
[13] W.-C. Wu and M.-S. Tsai, "Application of enhanced integer coded particle swarm optimization for distribution system feeder reconfiguration," IEEE Transactions on Power Systems, vol. 26, no. 1, pp. 1-9, 2011.

[14] L. Zhenkun et al., "A hybrid particle swarm optimization algorithm for distribution network reconfiguration," Proceedings of the Chinese Society of Electrical Engineering, vol. 28, no. 31, pp. 35-41, 2008.

[15] R. Huaipu, S. Siqing, and W. Xiaowei, "Based on the improved binary particle swarm optimization algorithm of network reconfiguration," Power Grid And Clean Energy and Infected, vol. 27, no. 8, pp. 41-43, 2011.

[16] V. Calderaro, A. Piccolo, and P. Siano, "Maximizing DG penetration in distribution networks by means of GA based reconfiguration," in Proceedings of the 2005 International Conference on Future Power Systems, Netherlands, November 2005.

[17] D. I. H. Sun, S. Abe, R. R. Shoults, M. S. Chen, P. Eichenberger, and D. Farris, "Calculation of energy losses in a distribution system," IEEE Transactions on Power Apparatus and Systems, vol. 99, no. 4, pp. 1347-1356, 1980.

[18] Z. Fang and C. S. Cheng, "A modified newton method for radial distribution system power flow analysis," IEEE Transactions on Power Systems, vol. 12, no. 1, pp. 389-397, 1997.

[19] W. Jincheng, W. Jiesheng, and W. Wang, "Parameter self-tuning PID control and decision based on Improved Particle Swarm Optimization (PSO)," 2005,20 (1):73-76.

[20] D. P. Bernardon, A. P. C. Mello, L. L. Pfitscher, L. N. Canha, A. R. Abaide, and A. A. B. Ferreira, "Real-time reconfiguration of distribution network with distributed generation," Electric Power Systems Research, vol. 107, pp. 59-67, 2014. 


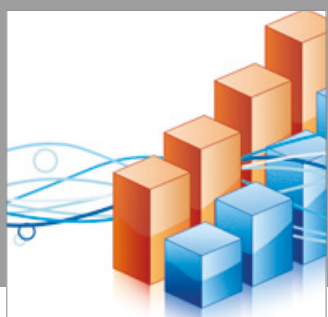

Advances in

Operations Research

vatersals

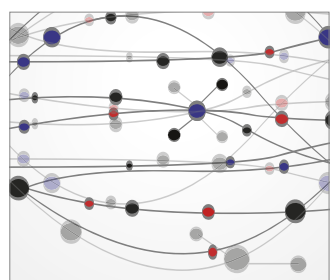

\section{The Scientific} World Journal
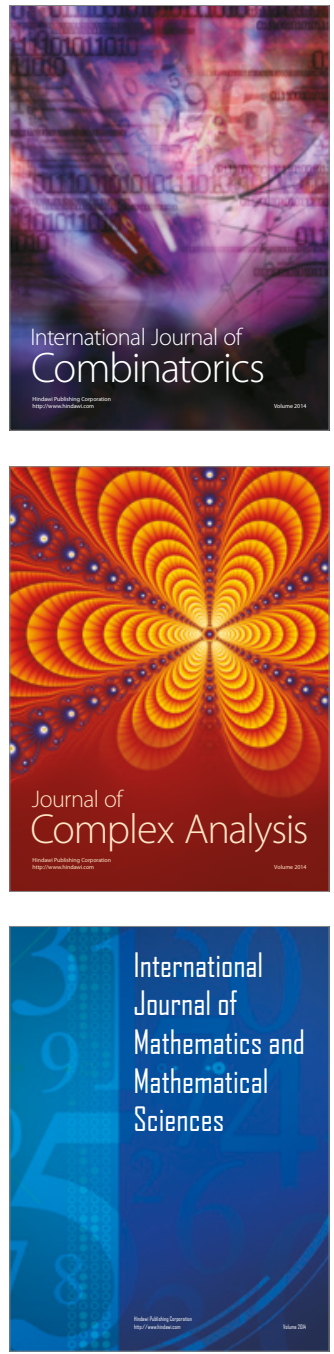
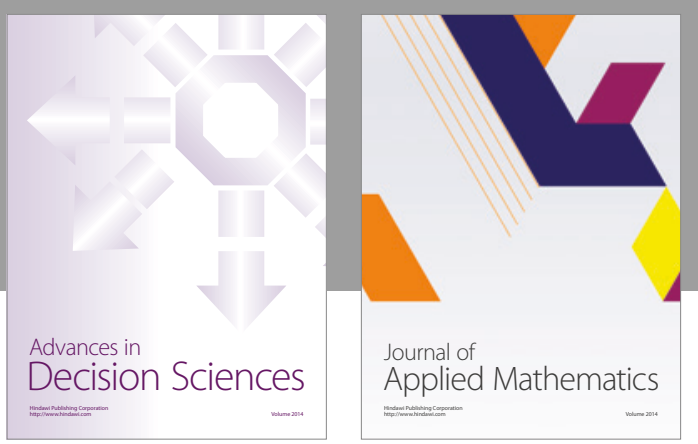

Algebra

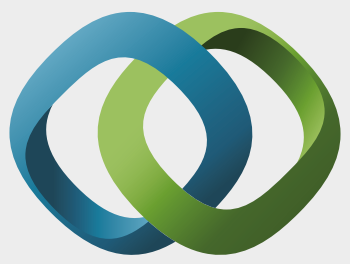

\section{Hindawi}

Submit your manuscripts at

https://www.hindawi.com
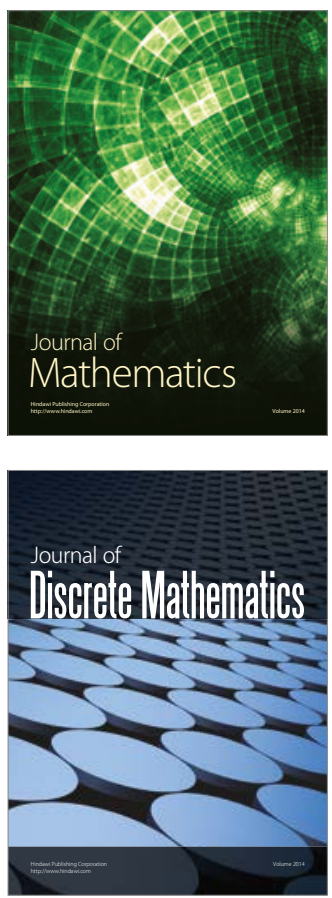

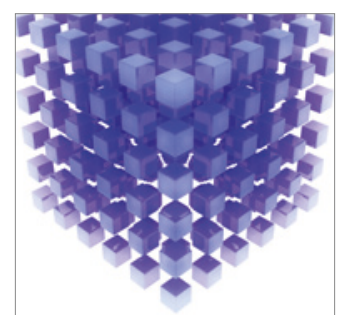

Mathematical Problems in Engineering
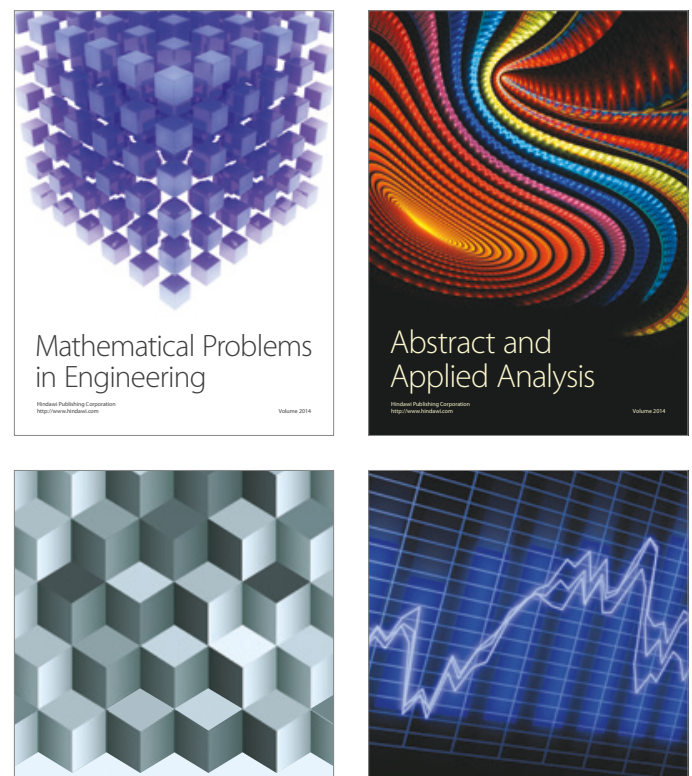

Journal of

Function Spaces

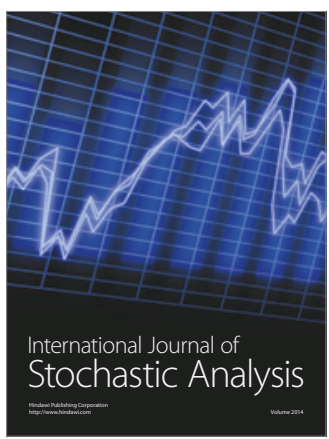

Probability and Statistics
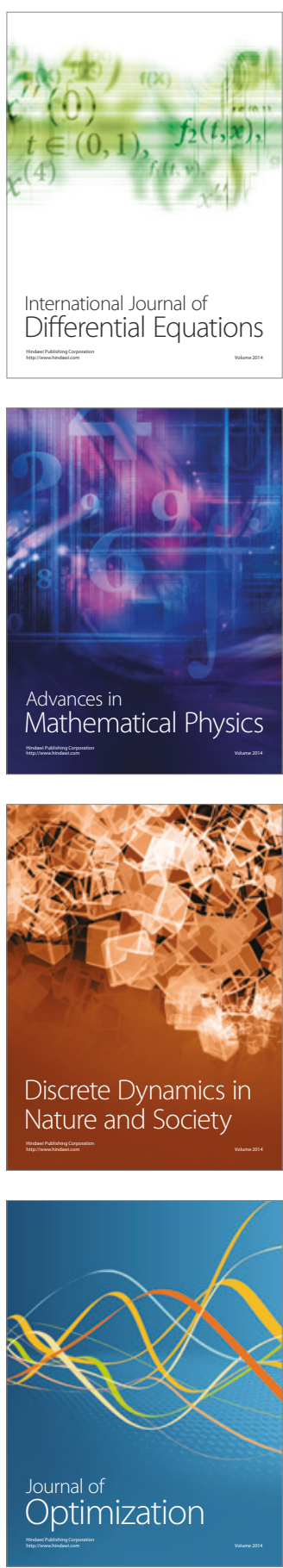